\title{
On Addressing the Empty Answer Problem in Uncertain Knowledge Bases
}

\author{
Ibrahim Dellal, Stéphane Jean, Allel Hadjali, Brice Chardin, Mickaël Baron \\ LIAS/ISAE-ENSMA - University of Poitiers \\ 1, Avenue Clement Ader, 86960 Futuroscope Cedex, France \\ \{firstname. lastname\}@ensma.fr
}

\begin{abstract}
Recently, several large Knowledge Bases (KBs) have been constructed by mining the Web for information. As an increasing amount of inconsistent and non-reliable data are available, KBs facts may be uncertain and are then associated with an explicit certainty degree. When querying these uncertain KBs, users seek high quality results i.e., results that have a certainty degree greater than a given threshold $\alpha$. However, as they usually have only a partial knowledge of the KBs contents, their queries may be failing i.e., they return no result for the desired certainty. To prevent this frustrating situation, instead of returning an empty set of answers, our approach explains the reasons of the failure with a set of $\alpha$ Minimal Failing Subqueries ( $\alpha \mathrm{MFSs}$ ), and computes alternative relaxed queries, called $\alpha$ MaXimal Succeeding Subqueries ( $\alpha \mathrm{XSSs}$ ), that are as close as possible to the initial failing query. Moreover, as the user may not always be able to provide an appropriate threshold $\alpha$, we propose two algorithms to compute the $\alpha$ MFSs and $\alpha$ XSSs for other thresholds. Our experiments on the WatDiv benchmark show the relevance of our algorithms compared to a baseline method.
\end{abstract}

\section{Introduction}

A Knowledge Base (KB) is a collection of entities and facts about them. Wellknown examples of KBs include Knowledge Vault [1] and YAGO [2]. These KBs contain billions of facts captured as RDF triples (subject, predicate, object) and are queried with the SPARQL language. As these KBs have been constructed by mining the Web for information, their facts are uncertain (i.e., potentially inconsistent). Therefore, an explicit degree of certainty is assigned to KB facts. When querying uncertain KBs, users expect to obtain high quality results i.e., results that have a certainty degree greater than a given threshold $\alpha$. However, as they rarely know the underlying structure and contents of a KB, they may be faced with the empty answer problem i.e., they obtain no result or results with a degree of certainty lower than $\alpha$. This is not an uncommon problem. Indeed, the study conducted by Saleem et al. on SPARQL endpoints shows that ten percent of queries submitted to DBpedia between May and July 2010 returned empty results [3]. Instead of solely returning an empty set as the answer of a query, the system might help the user understand the reasons of this failure by providing 
him/her with a set of Minimal Failing Subqueries (MFSs). Moreover, interesting non-failing relaxed queries, called Maximal Succeeding Subqueries (XSSs), might be suggested to the user by the system as well.

The problem of computing MFSs and XSSs of SPARQL queries expressed on KBs has already been addressed by Fokou et al. [4]. In this paper, we consider a generalization of MFSs and XSSs in the context of uncertain KBs. We call $\alpha$ MFSs and $\alpha$ XSSs the failure causes and maximal relaxed subqueries of a query that filters results according to their certainty degree and a given threshold $\alpha$. We first show under which conditions the computation of MFSs and XSSs can be directly adapted to $\alpha \mathrm{MFSs}$ and $\alpha \mathrm{XSSs}$. In this setting, the user has to define the threshold $\alpha$. However, as she/he may not have an idea of the certainty degrees assigned to the KB RDF triples, we also investigate the idea of suggesting relaxed queries with lower $\alpha$ thresholds. This kind of relaxation requires the computation of $\alpha \mathrm{MFSs}$ and $\alpha \mathrm{XSSs}$ for multiple thresholds $\alpha$. To save computation time, some properties between $\alpha \mathrm{MFSs}$ and $\alpha \mathrm{XSSs}$ of different thresholds are established and exploited. Thus, depending on which order the $\alpha$ values are considered, two approaches Top-Down and Bottom-Up are discussed. We run the experiments on the WatDiv benchmark with the Jena TDB quadstore to show the impact of our approaches.

The paper is structured as follows. Section 2 formalizes the problem. Section 3 defines the conditions under which a previous work algorithm can be directly adapted to find the $\alpha$ MFSs and $\alpha$ XSSs for a given $\alpha$. Section 4 describes the two proposed approaches to compute $\alpha$ MFSs and $\alpha$ XSSs for a set of thresholds. Section 5 discusses the experimental evaluation performed. Section 6 details related work and Section 7 concludes.

\section{Problem statement}

An RDF triple is a triple (subject, predicate, object) $\in(U \cup B) \times U \times(U \cup B \cup L)$ where $U$ is a set of URIs, $B$ is a set of blank nodes and $L$ is a set of literals. We denote by $T$ the union $U \cup B \cup L$. An RDF database (or triplestore) is a set of $R D F$ triples (denoted by $T_{R D F}$ ). Each RDF triple has a trust score representing the trustworthiness of the triple. This score is assigned with the function $t v: T_{R D F} \rightarrow[0,1]$.

An $R D F$ triple pattern $t$ is a triple (subject, predicate, object) $\in(U \cup V) \times$ $(U \cup V) \times(U \cup V \cup L)$, where $V$ is a set of variables disjoint from the sets $U, B$ and $L$. We denote by $\operatorname{var}(t) \subseteq V$ the set of variables occurring in $t$. We consider RDF queries defined as a conjunction of triple patterns: $Q=t_{1} \wedge \cdots \wedge t_{n}$. The number of triple patterns of a query $Q$ is denoted by $|Q|$ and its variables $\operatorname{var}(Q)=\bigcup \operatorname{var}\left(t_{i}\right)$.

A mapping $\mu$ from $V$ to $T$ is a partial function $\mu: V \rightarrow T$. For a triple pattern $t$, we denote by $\mu(t)$ the triple obtained by replacing in $t$ its variables $\operatorname{var}(t)$ by their mapping $\mu(\operatorname{var}(t))$. Let $D$ be an $R D F$ database, $t$ a triple pattern. The evaluation of the triple pattern $t$ over $D$ denoted by $[[t]]_{D}$ is defined by: $[[t]]_{D}=\{\mu \mid \operatorname{dom}(\mu)=\operatorname{var}(t) \wedge \mu(t) \in D\}$. Let $Q$ be a query, the evaluation of $Q$ 
over $D$ is defined by: $[[Q]]_{D}=\left[\left[t_{1}\right]\right]_{D} \bowtie \cdots \bowtie\left[\left[t_{n}\right]\right]_{D}$. Let $\mu$ be a solution of the query $Q=t_{1} \wedge \cdots \wedge t_{n}$ and aggreg be an aggregation function (e.g, the minimum), the trust value of $\mu$ is defined by $t v(\mu, Q)=\operatorname{aggreg}\left(t v\left(\mu\left(t_{1}\right)\right), \cdots, t v\left(\mu\left(t_{n}\right)\right)\right)$. The evaluation of $Q$ over $D$ that returns trust weighted results with a threshold $\alpha$ is defined by: $[[Q]]_{D}^{\alpha}=\left\{\mu \in[[Q]]_{D} \mid t v(\mu) \geq \alpha\right\}$.

Given a query $Q=t_{1} \wedge \cdots \wedge t_{n}$, a query $Q^{\prime}=t_{i} \wedge \cdots \wedge t_{j}$ is a subquery of $Q$, $Q^{\prime} \subseteq Q$, iff $\{i, \cdots, j\} \subseteq\{1, \cdots, n\}$. If $\{i, \cdots, j\} \subset\{1, \cdots, n\}$, we say that $Q^{\prime}$ is a proper subquery of $Q\left(Q^{\prime} \subset Q\right)$. An $\alpha$ Minimal Failing Subquery $(\alpha \mathrm{MFS}) Q^{*}$ of a query $\mathrm{Q}$ for a given $\alpha$ is defined by: $\left[\left[Q^{*}\right]\right]_{D}^{\alpha}=\emptyset \wedge \nexists Q^{\prime} \subset Q^{*}$ such that $\left[\left[Q^{\prime}\right]\right]_{D}^{\alpha}=$ $\emptyset$. The set of all $\alpha$ MFSs of a query $Q$ for a given $\alpha$ is denoted by $m f s^{\alpha}(Q)$. An $\alpha$ Maximal Succeeding Subquery ( $\alpha \mathrm{XSS}) Q^{*}$ of a query Q for a given $\alpha$ is defined by: $\left[\left[Q^{*}\right]\right]_{D}^{\alpha} \neq \emptyset \wedge \nexists Q^{\prime}$ such that $Q^{*} \subset Q^{\prime} \wedge\left[\left[Q^{\prime}\right]\right]_{D}^{\alpha} \neq \emptyset$. The set of all $\alpha \mathrm{XSSs}$ of a query $Q$ for a given $\alpha$ is denoted by $x \operatorname{ss}^{\alpha}(Q)$.

Problem statement. We are concerned with computing $m f s^{\alpha_{i}}(Q)$ and $x s^{\alpha_{i}}(Q)$ of a failing $R D F$ query $Q$ for a set of thresholds $\left\{\alpha_{1}, \cdots, \alpha_{n}\right\}$.

\section{$3 \alpha$ MFSs and $\alpha$ XSSs computation for a single $\alpha$}

In this section, we first give a direct adaptation of the Lattice-Based Approach (LBA) proposed in [4] to compute the $\alpha$ MFSs and $\alpha$ XSSs of a query for a given $\alpha$. It has the same algorithmic complexity as LBA (detailed in [4]). $\alpha$ LBA explores the lattice of subqueries by following a three-steps procedure.

1. Find an $\alpha$ MFS $Q^{*}$ of $Q$. Following Algorithm $1, \alpha$ LBA removes iteratively each triple pattern $t_{i}$ from $Q$, resulting in the proper subquery $Q^{\prime}$. If $Q^{\prime}$ fails for $\alpha$, then $Q^{\prime}$ contains an $\alpha$ MFS. Conversely, if $Q^{\prime}$ succeeds, then each $\alpha$ MFS of $Q$ contains $t_{i}$. The proof of this property relies on the fact that a successful query cannot contain a failing query [4].



2. Compute potential $\alpha$ XSSs i.e., the maximal queries that do not include the $\alpha$ MFS previously found. The set of potential $\alpha X S S s$ is denoted by $\operatorname{pxss}\left(Q, Q^{*}\right)$. This set can be computed as follows:

$$
\operatorname{pxss}\left(Q, Q^{*}\right)= \begin{cases}\emptyset, & \text { if }|Q|=1 . \\ \left\{Q-t_{i} \mid t_{i} \in Q^{*}\right\}, & \text { otherwise. }\end{cases}
$$


3. Test potential $\alpha$ XSSs. If a subquery found during step 2 succeeds, it is then an $\alpha$ XSS. If it fails, we apply the two previous steps on this particular subquery to find a new $\alpha$ MFS and its associated potential $\alpha$ XSSs. This is illustrated by Algorithm 2. It is worth noting that this algorithm includes mechanisms to avoid discovering the same $\alpha$ MFSs multiple times (lines 11-13).

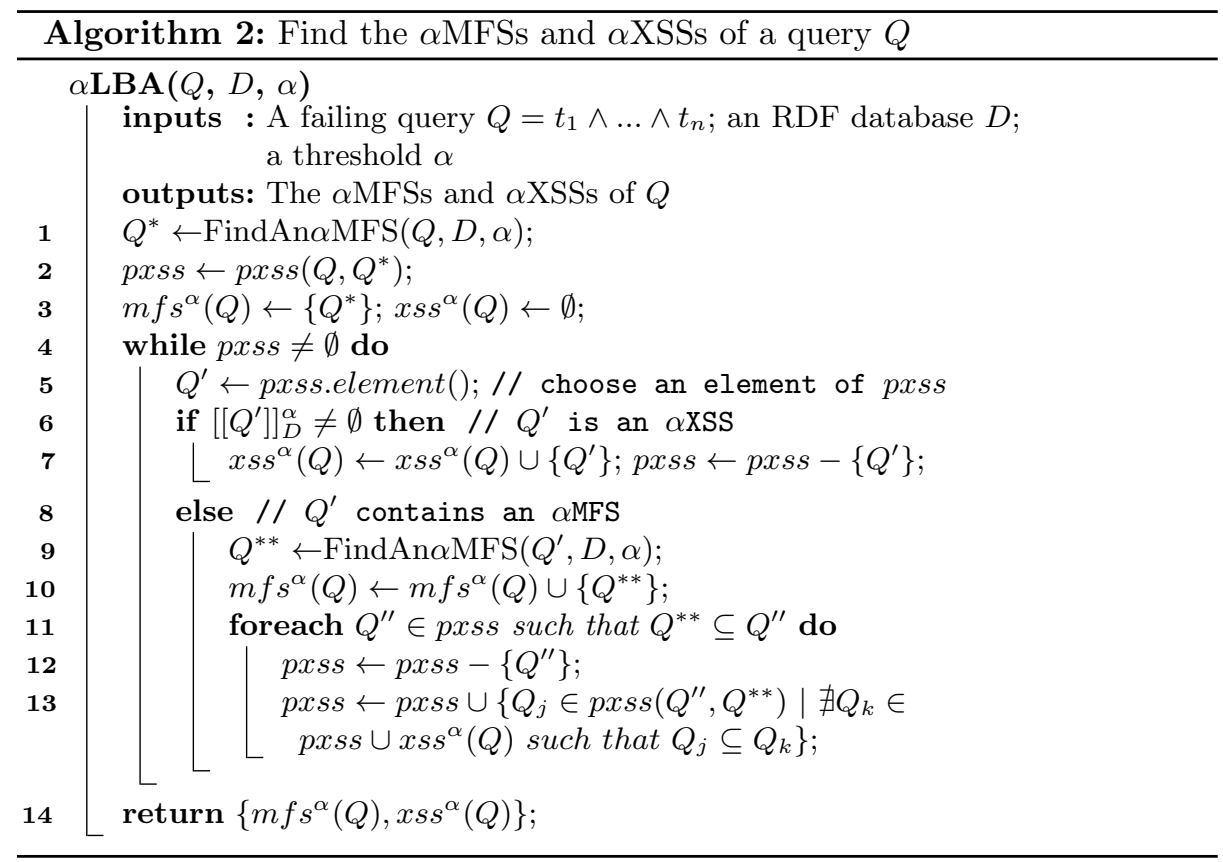

The $\alpha$ LBA algorithm relies on the fact that a successful query cannot contain a failing query. In the context of uncertain KBs, this property does not always hold depending on the chosen trust value aggregate function (aggreg). For example, with the maximum aggregate function, the degree of certainty of results potentially increases with additional triple patterns. Thus, a query may be failing but not its subqueries. The algorithm $\alpha$ LBA can only be used if the aggregate function aggreg is monotonic decreasing with respect to the subset partial order. We omit the proof of this property.

Definition 1. Let aggreg $:[0,1]^{n} \rightarrow[0,1]$ be an aggregate function, aggreg is monotonic decreasing with respect to set ${ }^{1}$ inclusion if for all sets $A$ and $B$ $\in[0,1]^{n}, A \subseteq B \Rightarrow \operatorname{aggreg}(A) \geq \operatorname{aggreg}(B)$.

As examples of monotonic decreasing aggregate functions, we can cite the minimum or the product restricted to values $\in[0,1]$.

\footnotetext{
${ }^{1}$ For simplicity, this definition is restricted to sets but could be extended to multisets.
} 
Proposition 1. Let aggreg be monotonic decreasing. $[[Q]]_{D}^{\alpha}=\emptyset \wedge Q^{\prime} \subset Q \Rightarrow$ $\left[\left[Q^{\prime}\right]\right]_{D}^{\alpha}=\emptyset$. That is to say, if a proper subquery $Q^{\prime}$ of $Q$ fails for a given $\alpha$ (using the aggreg function) then $Q$ also fails for $\alpha$.

\section{$4 \alpha$ MFSs and $\alpha$ XSSs computation for a set of $\alpha$}

To find $\alpha$ MFSs and $\alpha$ XSSs for a set of $\alpha:\left\{\alpha_{1}, \cdots \alpha_{n}\right\}$, the $\alpha$ LBA algorithm can be applied for each $\alpha_{i}$. This baseline method is named NLBA. In this section, we discuss various improvements of this approach. The idea is that the $\alpha$ MFSs and $\alpha$ XSSs for a given threshold provide a set of hints to deduce some $\alpha$ MFSs and $\alpha$ XSSs with higher (or lower) thresholds.

\subsection{Bottom-Up Approach}

In this section, we consider two thresholds $\alpha_{i}$ and $\alpha_{j}$ such that $\alpha_{i}<\alpha_{j}$. If $Q^{*}$ is an $\alpha_{i}$ MFS of the query $Q$, then $Q^{*}$ also fails for $\alpha_{j}$. However, this subquery is not necessarily minimal for $\alpha_{j}$ and therefore might not be an $\alpha_{j}$ MFS. The following proposition provides a condition under which an $\alpha_{i}$ MFS is also an $\alpha_{j}$ MFS. Due to space constraints, proofs are omitted.

Proposition 2. Let $\alpha_{i}$ and $\alpha_{j}$ be two thresholds such that $\alpha_{i}<\alpha_{j}$ and $Q^{*}$ be an $\alpha_{i} M F S$ of $Q$ on a dataset D. If $\left|Q^{*}\right|=1$, then $Q^{*}$ is also an $\alpha_{j} M F S$ of $Q$.

As pointed out previously, for a subquery $Q^{*}$ to be an $\alpha_{j}$ MFS of a query $Q$, all its proper subqueries have to succeed. As stated in proposition 2, this property is always true if the query contains a single triple pattern. Checking if a query has a single triple pattern does not require any database access. Thus, this case is checked first and all discovered $\alpha_{j}$ MFS of $Q$ are put in a set of discovered $\alpha M F S s$ denoted by $d m f s^{\alpha_{j}}(Q)$. Otherwise, proving that $Q^{*}$ is an $\alpha_{j}$ MFS requires checking that all its subqueries succeed, by executing those $\left|Q^{*}\right|$ queries. In the worst case where $Q^{*}$ is not an $\alpha_{j}$ MFS, $\left|Q^{*}\right|$ queries are executed without finding any $\alpha_{j}$ MFS. Conversely, the algorithm FindAn $\alpha$ MFS of $\alpha \mathrm{LBA}$ (Algorithm 1) also requires $\left|Q^{*}\right|$ queries but guarantees that an $\alpha$ MFS will be found. Thus, our approach favors FindAn $\alpha$ MFS over executing the subqueries of the $\alpha_{i}$ MFS to discover new $\alpha_{j}$ MFSs, as shown in Algorithm 3.

As for the $\alpha$ XSSs, an $\alpha_{i}$ XSS of $Q$ may fail for $\alpha_{j}$. The following proposition shows that if it succeeds, it is then an $\alpha_{j}$ XSS of $Q$.

Proposition 3. Let $\alpha_{i}$ and $\alpha_{j}$ be two thresholds such that $\alpha_{i}<\alpha_{j}$ and $Q^{*}$ be an $\alpha_{i} X S S$ of $Q$ on a dataset D. If $\left[\left[Q^{*}\right]\right]_{D}^{\alpha_{j}} \neq \emptyset$, then $Q^{*}$ is an $\alpha_{j} X S S$ of $Q$.

Thus, discovering if an $\alpha_{i} \mathrm{XSS}$ is also an $\alpha_{j} \mathrm{XSS}$ only requires the execution of a single query $\left(\alpha_{i}\right.$ XSS with the new threshold $\left.\alpha_{j}\right)$. This enables us to find a set of discovered $\alpha_{j} \mathrm{XSSs}$, denoted $d x s s^{\alpha_{j}}(Q)$.

Algorithm 3 presents our complete approach to find some $\alpha_{j}$ MFSs and $\alpha_{j}$ XSSs from the set of $\alpha_{i}$ MFSs and $\alpha_{i}$ XSSs. All $\alpha_{i}$ MFSs that have one triple pattern 
(oneAtom) are inserted in $d m f s^{\alpha_{j}}(Q)$ (line 1). Then, the algorithm iterates over the $\alpha_{i}$ MFSs with at least two triple patterns (the set $F Q$ ). It searches an $\alpha_{j}$ MFS $Q^{*}$ in a query $Q^{\prime}$ of $F Q$ with the FindAn $\alpha$ MFS algorithm (line 5). Then, it removes all the failing queries of $F Q$ that contain $Q^{*}$ since they cannot be minimal. This process stops when all the queries in $F Q$ have been processed (they have either been used to find an $\alpha_{j}$ MFS or removed as they contain a found $\alpha_{j}$ MFS). Some $\alpha_{j}$ XSSs are then identified simply by executing each $\alpha_{i}$ XSS and keeping those that are succeeding (lines 9-10).

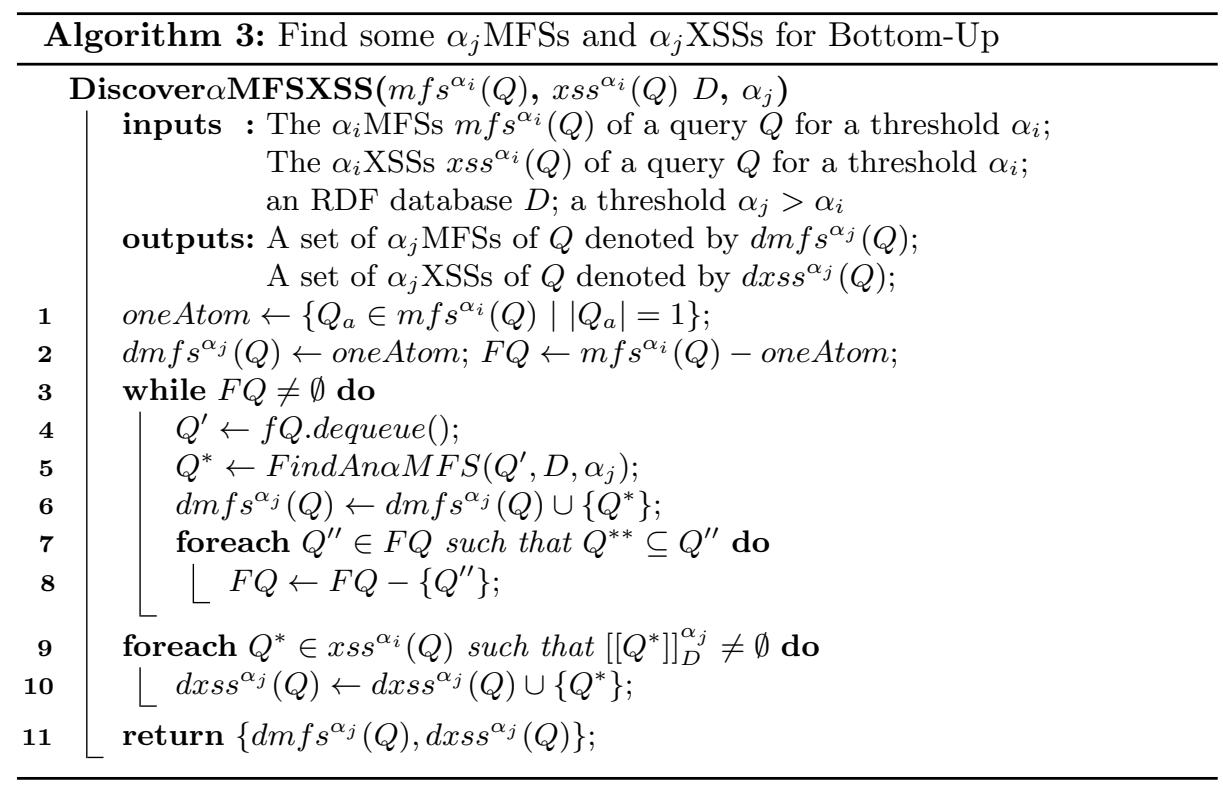

Once some $\alpha_{j}$ MFSs and $\alpha_{j}$ XSSs have been discovered, an optimized version of $\alpha \mathrm{LBA}$ is executed that takes these discovered $\alpha_{j}$ MFSs and $\alpha_{j}$ XSSs as inputs, then computes the remaining $\alpha_{j}$ MFSs and $\alpha_{j}$ XSSs.

\subsection{Top-Down Approach}

We also consider a Top-Down approach that computes the $\alpha$ MFSs and $\alpha$ XSSs using threshold values in descending order. Thanks to the duality relation that holds between $\alpha$ MFS and $\alpha$ XSS, the properties used in this approach are dual to the ones used in the bottom-up approach.

\section{Experimental Evaluation}

Here we investigate the scalability of our approaches and compare them with the baseline method $N L B A$ (executing $\alpha$ LBA for each of the $N$ thresholds). 
Experimental Setup. We have implemented the proposed algorithms in JAVA 1.864 bits. In our current implementation, these algorithms are run on top of Jena TDB. We chose Jena TDB because it is a quadstore that allows us to store the degree of certainty for each triple. Moreover, Jena TDB provides a low level quad filter hook ${ }^{2}$ that we use to retrieve results satisfying the provided threshold. Our implementation is available at https://forge.lias-lab.fr/projects/qars4ukb with a tutorial to reproduce our experiments.

Our experiments were conducted on a Ubuntu Server 16.04 LTS system with Intel XEON CPU E5-2630 v3 @2.4Ghz CPU and 16GB RAM. For our experiments, we chose the min aggregate function.

Dataset and Queries. We used a dataset of $20 \mathrm{M}$ triples generated with the WatDiv benchmark [5]. The certainty degree of each RDF triple were generated randomly. We consider 7 failing queries ${ }^{3}$. These queries range between 1 and 15 triple patterns and cover the main query patterns: star (characterized by subjectsubject joins between triple patterns), chain (composed of object-subject joins) and composite (made of other join patterns).

Experiment. Figure 1 shows the execution times of each algorithm for each query on Jena TDB with the 20M triples dataset. Figure 2 gives the number of executed queries by each algorithm. This experiment has been run with the thresholds arbitrarily set to $\{0.2,0.4,0.6,0.8\}$. In comparison with $N L B A$, our algorithms execute fewer queries for finding the $\alpha \mathrm{MFSs}$ and $\alpha \mathrm{XSSs}$ of each workload query. Overall, Bottom-Up and Top-Down execute respectively $39 \%$ and $44 \%$ fewer queries than $N L B A$. As a consequence, these algorithms have shorter execution times (a decrease of respectively $30 \%$ and $42 \%$ execution times for Bottom-Up and Top-Down). For some queries, this improvement is important. For example, $N L B A$ needs 7 seconds to find the $\alpha$ MFSs and $\alpha$ XSSs of the query Q2, whereas our algorithms need around 1 second. Execution times depend heavily on the queries that our algorithms avoid executing. For example, our algorithms execute around 30 queries for Q4 whereas $N L B A$ needs 120 queries. For Top-Down, this result is an important performance gain $94 \%$. This is not the case for Bottom-Up that has nearly the same execution time than NLBA. By analyzing the executed queries, we find that Bottom-Up prevents the execution of queries that have short execution times but keeps the most expensive ones. Thus, the overall execution time is almost unchanged.

This experiment also shows that none of our algorithms provides the best result for every query. Bottom-Up offers the best execution times for Q1, Q2 and Q5 whereas Top-Down is the most efficient for Q3, Q4 and Q6. Despite executing the least number of queries, Bottom-Up does not offer the best total execution time for this workload. Conversely, Top-Down executes the greatest number of queries but has the best execution time. This is due to the fact that our algorithm executes different queries that have different execution times. In particular, Top-Down starts by searching the $\alpha$ MFSs and $\alpha$ XSSs for the highest thresholds. The executed queries tend to be selective as the threshold is high

\footnotetext{
${ }^{2}$ http://jena.apache.org/documentation/tdb/quadfilter.html

${ }^{3}$ available at https://forge.lias-lab.fr/projects/qars4ukb/wiki/Doc\#Queries
} 


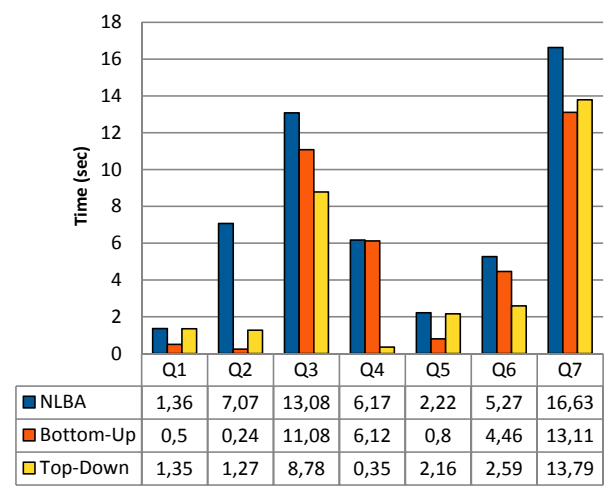

Fig. 1. Execution time (20M triples)

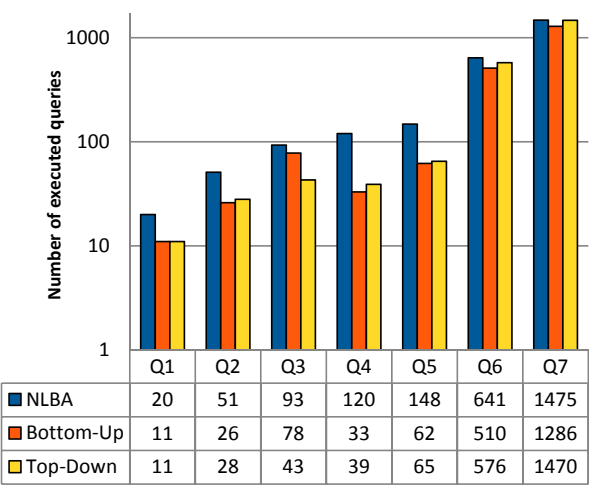

Fig. 2. \# Executed queries (log scale)

and thus, they have short execution times. Once the $\alpha$ MFSs and $\alpha$ XSSs for the highest thresholds are found, they avoid the execution of queries with a lower threshold that are likely to be more expensive. As Bottom-Up follows the dual approach, it tends to execute non-selective queries and has the overall worst performance.

\section{Related Work}

Several approaches proposed relaxation operators in the RDF context. These operators are mainly based on RDFS semantics [6-8], similarity measures [9, 10] and user preferences [11]. They generate a set of relaxed queries, ordered by similarity with the original query and executed in this order $[6,7,12]$. Relaxation operators are directly used by the user in her/his query [8] or combined with query rewriting rules to perform relaxation [11]. In these approaches, the failure causes of the query are unknown, which may lead to executing unnecessary relaxed queries. Fokou et al. [4] tackled this problem by defining the LBA and MBA approaches to compute the MFSs and XSSs of the query. Our approach is based on the LBA algorithm. We have extended this work by identifying the condition under which LBA can be used in the context of uncertain KBs and by defining two algorithms to compute $\alpha \mathrm{MFSs}$ and $\alpha$ XSSs for several thresholds. Our work is among the pioneering works aiming at exploring the query relaxation issue in uncertain KBs. To the best of our knowledge, the only other work in this context is [12]. However, this work only uses the trust value to order results by their trustworthiness. They do not consider, as we do in this paper, queries that return no result satisfying the provided trust threshold.

\section{Conclusion}

In this paper, we have considered the empty answer problem in the context of uncertain KBs. To provide the user with a relevant feedback, we have proposed 
to compute the $\alpha$ MFSs and $\alpha$ XSSs of the failing query as they give a clear overview of the query failure causes and a set of relaxed queries that she/he can execute to find some useful alternative answers. We have first defined the condition under which a previous work algorithm can be directly adapted to the context of uncertain KBs. Then, we have studied the problem of computing the $\alpha$ MFSs and $\alpha$ XSSs for multiple thresholds by defining two approaches that consider $\alpha$ thresholds in different orders. We have done a complete implementation of these algorithms and shown experimentally on WatDiv benchmark that our approaches outperform the baseline method.

In our experiments, none of our algorithms has the best performance for all queries. As a future work, we plan to study the conditions under which an algorithm may provide the best results. An analysis of the queries executed by our algorithms shows that they share some triple patterns. Thus, we will investigate multiple-query optimization techniques to further improve their execution times.

\section{References}

1. Dong, X., Gabrilovich, E., Heitz, G., Horn, W., Lao, N., Murphy, K., Strohmann, T., Sun, S., Zhang, W.: Knowledge Vault: A Web-scale Approach to Probabilistic Knowledge Fusion. In: KDD'14. (2014) 601-610

2. Hoffart, J., Suchanek, F.M., Berberich, K., Weikum, G.: YAGO2: A Spatially and Temporally Enhanced Knowledge Base from Wikipedia. Artificial Intelligence 194 (2013) 28-61

3. Saleem, M., Ali, M.I., Hogan, A., Mehmood, Q., Ngomo, A.N.: LSQ: The Linked SPARQL Queries Dataset. In: ISWC'15. (2015) 261-269

4. Fokou, G., Jean, S., Hadjali, A., Baron, M.: Handling Failing RDF Queries: From Diagnosis to Relaxation. Knowledge and Information Systems (KAIS) 50(1) (2017)

5. Aluç, G., Hartig, O., Özsu, M.T., Daudjee, K.: Diversified Stress Testing of RDF Data Management Systems. In: ISWC'14. (2014) 197-212

6. Hurtado, C.A., Poulovassilis, A., Wood, P.T.: Ranking Approximate Answers to Semantic Web Queries. In: ESWC'09. (2009) 263-277

7. Huang, H., Liu, C., Zhou, X.: Approximating query answering on RDF databases. Journal of the World Wide Web: Internet and Web Information Systems (WWW) 15(1) (2012) 89-114

8. Calí, A., Frosini, R., Poulovassilis, A., Wood, P.: Flexible Querying for SPARQL. In: ODBASE'14. (2014) 473-490

9. Hogan, A., Mellotte, M., Powell, G., Stampouli, D.: Towards Fuzzy Queryrelaxation for RDF. In: ESWC'12. (2012) 687-702

10. Elbassuoni, S., Ramanath, M., Weikum, G.: Query Relaxation for EntityRelationship Search. In: ESWC'11. (2011) 62-76

11. Dolog, P., Stuckenschmidt, H., Wache, H., Diederich, J.: Relaxing RDF queries based on user and domain preferences. Journal of Intelligent Information Systems (JIIS) 33(3) (2009) 239-260

12. Reddy, K.B., Kumar, P.S.: Efficient Trust-Based Approximate SPARQL Querying of the Web of Linked Data. In: Uncertainty Reasoning for the Semantic Web II. Springer (2013) 315-330 